\title{
What's New in Neurosurgery: Advances in Neurovascular and Spine Surgery, Epilepsy Surgery, Surgery for Movement Disorders and Intraoperative Imaging
}

\author{
George J. Dohrmann Richard W. Byrne \\ Section of Neurosurgery, University of Chicago Medical Center and Department of Neurological Surgery, \\ Rush University Medical Center, Chicago, III., USA
}

Curing problems of the brain without manipulating the brain or without much manipulation of the brain has been the goal of neurosurgeons for decades. Difficult problems such as aneurysms and arteriovenous malformations were dealt with by operating on the cerebral vessels. With the improved technology it now is possible in many patients to block selectively with microcatheters such threatening vascular problems using endovascular coils (or endovascular stents) or injection of substances via the microcatheters to selectively obstruct vessels within the brain. All of this can be done without open operations on the brain.

Ischemic stroke was thought to be a condition simply to be diagnosed but not treated. Now, depending upon the time of occurrence, the thrombosed vessel may be treated by antithrombolytic agents and/or microcatheter techniques to remove the thrombus/embolus and insert a microstent to keep the artery patent. Endovascular neurosurgeons have been very active in this area. In some medical centers, these strokes are called 'brain attacks' (like myocardial infarction is called 'heart attack') to emphasize the need to treat them aggressively and early, if possible.

Treatment of spine disorders has undergone a renaissance. Now $75-85 \%$ of most neurosurgical practice in the
United States involves the diagnosis and treatment of spine problems. Numerous types of spine instrumentation are available to do spinal fusions. Minimally invasive spine techniques are available, allowing patients having various spine operations to return home the same day. The role, if any, of the artificial disc is being evaluated. There are attempts to close the hole in the annulus of the disc in patients having an operation for disc herniation to assess whether the risk of recurrent disc herniation is decreased. Spacers are being evaluated that are placed between the spinous processes to prevent lordosis in patients with spinal stenosis who have compression of spinal cord and/or nerve roots with extension of the spine. In neurosurgery, in general, and in spine surgery, in particular, the pace of technical advances often has been outpacing demonstrable clinical efficacy.

A significant resurgence in the surgical treatment of epilepsy has followed a series of publications over the last 10 years. Wiebe et al. [1] prospectively studied patients with mesial temporal sclerosis, the most common cause of adult intractable epilepsy, and reported their findings in the New England Journal of Medicine. Their publication was the first prospective, randomized study to show definitely that epilepsy surgery was superior to best medical therapy in medically intractable temporal lobe epi-

\section{KARGER}

Fax +4161306 1234

E-Mail karger@karger.ch

www.karger.com
(C) 2010 S. Karger AG, Basel

$1011-7571 / 10 / 0195-0328 \$ 26.00 / 0$

Accessible online at:

www.karger.com/mpp
Prof. George J. Dohrmann

Section of Neurosurgery, University of Chicago Medical Center and Department of Neurological Surgery, Rush University Medical Center Chicago, IL 60637 (USA)

Tel. +1 773702 2123, Fax +1 7737023518 
lepsy. In their series, $58 \%$ of patients in the epilepsy surgery group were seizure-free at 1 year while only $8 \%$ of patients in the best medical group therapy group were seizure-free $(\mathrm{p}<0.01)$. Complication rates were similar between the two groups. This publication, along with publications showing that epilepsy surgery was highly cost-effective, had a very low permanent complication rate and led to substantial improvements in quality of life, has caused neurologists and neurosurgeons to consider epilepsy a surgically remediable disease.

Deep brain stimulation has been used to treat epilepsy in patients with the seizure focus in eloquent cortex or where there are multiple seizure foci not amenable to resection. This treatment is helpful in those difficult situations.

The idea of therapeutic stimulation of the brain for chronic neurologic disorders was first attempted in movement disorders. Deep brain stimulation of the subthalamic nucleus was introduced as a last resort therapy for patients with severe Parkinson's disease as a reversible therapy to replace the older technique of selective lesioning of the globus pallidus. What was once considered an experimental technique is now supported by class I evidence of efficacy. Outcomes are similar to expected best outcomes of medical therapy, but without the disabling side effects of medical therapy. Extensions of deep brain stimulation treatment of movement disorders have led to successful treatment of both essential tremor and dystonia.
Another advance in cranial neurosurgery is in the field of intraoperative navigation and intraoperative imaging. Neuronavigation, the ability to use stereotactically registered pre-operative images during surgery to precisely locate lesions, has been around for more than 15 years. Neuronavigation is commonly found in operating rooms throughout the United States. A relatively small but growing number of institutions have the capability to do actual intraoperative imaging. Intraoperative MRI and CT scanners are being introduced with mixed reviews. Preliminary results have shown these devices to be of some use in brain tumor surgery to further assure a maximal safe resection.

Intraoperative CT scanning and portable CT scanners in the operating room have been used to identify landmarks more reliably and place spinal instrumentation. Investigations are being done currently to see if the use of this technology leads to less radiation exposure to the patient and to the operating room staff.

The advances in neurosurgery, partially summarized above, give an indication of why the past years have been a significant leap forward for the field of neurosurgery.

Reference

1 Wiebe S, Blume WT, Girvin JP, Eliasziw M: A randomized controlled trial of surgery for temporal-lobe epilepsy. N Engl J Med 2001; 345:311-318 